Cite this: J. Mater. Chem. A, 2013, 1, 9418

Received 14th February 2013

Accepted 28th March 2013

DOI: $10.1039 / \mathrm{c} 3 \operatorname{ta} 10674 h$

www.rsc.org/MaterialsA

\section{Salt and sugar: direct synthesis of high surface area carbon materials at low temperatures via hydrothermal carbonization of glucose under hypersaline conditions}

\author{
Nina Fechler, ${ }^{*}$ Stephanie-Angelika Wohlgemuth, Philipp Jäker \\ and Markus Antonietti
}

Hydrothermal carbonization of carbohydrates, here glucose as a model, in salt-water mixtures results in high surface area carbonaceous materials with surface areas up to $650 \mathrm{~m}^{2} \mathrm{~g}^{-1}$ where porosity is created by aggregation of very small primary nanoparticles, similar to aerogels or high surface area soot. These materials can be obtained by simple washing with water and are useful without further activation processes. Furthermore, no special technical equipment for isolation is needed since the materials are exceptionally stable throughout vacuum drying, thus keeping the overall approach sustainable and very simple.

\section{Introduction}

Hydrothermal carbonization (HTC) is a process which can simplistically be described as the technical acceleration of natural biomass coalification on a timescale of hours and days rather than millions of years. Importantly, cheap and readily available precursors, e.g. biowaste or simple carbohydrates, can be turned into valuable carbonaceous materials by HTC., ${ }^{1,2}$ Among these materials, carbonaceous aerogels can be made, which are lightweight, nanostructured materials and are already widely applied in sorption, catalysis, as insulators, and as electrode materials. ${ }^{3-5}$

Since the first reports on silica aerogels in $1931,{ }^{6}$ aerogels have been prepared from many materials, such as metal oxides, alumina or metal chalcogens. ${ }^{5,7-9}$ In 1989 , the first organic aerogels were reported by Pekala et al. which were formed by the condensation of resorcinol-formaldehyde (RF) in the presence of acid or base catalysts. The resulting aerogels still contain abundant oxygen functionalities and are therefore classified as organic aerogels, yet they may be converted into carbon aerogels via pyrolysis. ${ }^{6}$ Furthermore, the addition of e.g. melamineformaldehyde into the RF-system was presented as a suitable

Max-Planck-Institute of Colloids and Interfaces, Department of Colloid Chemistry, Am Mühlenberg 1, 14424 Potsdam-Golm, Germany.E-mail: nina.fechler@mpikg.mpg.de method to synthesize nitrogen-doped aerogels. ${ }^{10,11}$ Essentially, carbon aerogels nicely complement their inorganic counterparts owing to properties such as chemical inertness and electrical conductivity which make them very attractive candidates for electrode materials or catalyst supports. ${ }^{12,13}$ Due to the high potential of these materials, the development of alternative and sustainable approaches towards such aerogels and low temperature porous carbons is of significant interest.

Our group has previously used various techniques to direct HTC towards useful high surface area carbonaceous materials (HSAC), e.g. using albumin as a gel-forming agent in combination with glucose. Within this approach nitrogen-doped carbon aerogels could be obtained, however, neither the nitrogen content nor the surface area was tunable. ${ }^{14}$ Using a different system, the borax-mediated hydrothermal carbonization of glucose, we were able to tune the particle size (and therefore the surface area) of the HSAC by varying the amount of borax added. ${ }^{15,16}$

Concerning simplicity and price, this already points to a potential next step, i.e. using commonly available salts (with sufficient polarizability and Lewis acid character) to stabilize the surface of the forming carbon materials in situ, keeping it from coagulation. Using very hydrophilic ions at the same time lowers the partial pressure of water and changes its structure so that reactions can potentially be performed under hypersaline but otherwise less extreme conditions as will be shown in the present work. This approach is also inspired by the salt-templating work carried out by our group. ${ }^{17}$ There, simple salts were used as templates to obtain valuable carbon materials using ionic liquids as precursors. Recycling of the reaction medium in all these cases is very simple: the salt is washed with water, filtered, and can be reused after evaporation of the water.

\section{Synthesis and methods}

\section{Materials and methods}

D-(+)-Glucose was purchased from Roth Chemicals. Lithium chloride, sodium chloride, potassium chloride and zinc 
chloride (99\%) and 2-pyrrol-carboxyaldehyde (PCA) were acquired from Sigma Aldrich. All chemicals were used without further purification.

The eutectic salt mixtures were freshly prepared prior to the synthesis by grinding and mixing the respective salts in the eutectic ratio: lithium chloride/zinc chloride (LiZ 23 mol\% lithium chloride), sodium chloride/zinc chloride (SZ $42 \mathrm{~mol} \%$ sodium chloride) and potassium chloride/zinc chloride (PZ 51 mol\% potassium chloride). Although technically not necessary, we stayed with eutectic salt mixtures to minimize the risk of potential crystallization under cooling, which would potentially destroy the as-formed carbon structure. It is however clear that also other than eutectic salt systems with Lewis acidic cations can be used.

In a typical synthesis, glucose ( $6 \mathrm{~g}$ ) was dissolved in varying amounts of deionized water. For the synthesis of the nitrogendoped carbon, PCA ( $1 \mathrm{~g})$ was added as a nitrogen source. The solution was thoroughly mixed with varying amounts of the respective salt (mixture), filled into a glass inlet and placed into a Teflon lined, stainless steel autoclave (45 l volume, Parr Instruments). The synthesis was conducted in a pre-heated furnace at $180{ }^{\circ} \mathrm{C}$ overnight. Afterwards, the autoclave was allowed to cool down to room temperature. In the case of $\mathrm{NaCl}$, the solid product was collected by vacuum filtration, washed with water and stirred in $1 \mathrm{l}$ of water overnight to remove the residual salt. The monolithic materials (made with $\mathrm{ZnCl}$ ) were first ground and then placed into 11 of water and stirred overnight. Afterwards, the samples were collected by vacuum filtration, washed with water and dried in a vacuum.

The resulting carbons (C) and the nitrogen-doped carbon (NC) are named according to the salt system (zinc chloride (Zn), lithium chloride (Li), sodium chloride $(\mathrm{Na})$ or potassium chloride $(\mathrm{K})$ ), with the numbers reflecting salt and water mass used in the precursor mixture. $\mathrm{C}-\mathrm{Zn9} / 3$ is thereby a pure carbon made with $9 \mathrm{~g}$ Zn salt, diluted with $3 \mathrm{~g}$ of water.

\section{Characterization}

Nitrogen sorption measurements were accomplished with $\mathrm{N}_{2}$ at $77 \mathrm{~K}$ after degassing the samples at $150{ }^{\circ} \mathrm{C}$ under vacuum for 20 hours using a Quantachrome Quadrasorb SI porosimeter. The apparent surface area was calculated by applying the BrunauerEmmett-Teller (BET) model to the isotherm data points of the adsorption branch in the relative pressure range $p / p_{0}<0.3$. TEM images were obtained using a Zeiss EM $912 \Omega$ instrument. SEM images were obtained on a LEO 1550-Gemini instrument after sputtering with platinum. Elemental analysis was accomplished as combustion analysis using a Vario Micro device.

\section{Results and discussion}

The basic concept of using hypersaline conditions is to stabilize the surface of the as-formed primary nanoparticles to avoid Ostwald ripening or excessive particle growth. These primary particles at sufficiently high concentrations (the percolation threshold) then undergo cross-linking towards the final porous materials. At intermediate concentrations, porous "soot" is obtained which is composed of interconnected particles, but is still dispersible as such. The more salt added, the smaller the primary particles are and hence the higher is the surface area.

In preliminary experiments, we found that neither water-free salts nor "hard salts", e.g. NaCl, show the expected influence on the sample morphology (Table 1, entries 1 and 2). On the other hand, we successfully introduced surface areas into the materials when the used salt ions were rather hygroscopic. At the same time, the salt mixtures had to contain some leftover water, both to ensure a liquid reaction medium and to enhance surface stabilization which is most probably provided via the hydration water. In contrast, highly diluted media do not result in any porous materials, thus confirming the prerequisite of a hypersaline environment as an advantageous reaction medium to form monolithic, aerogel-like carbons (Table 1, entry 5).

In this paper, we will focus on slightly aqueous zinc chloride melts, of which the crystallization tendency can be further lowered by addition of small amounts of $\mathrm{LiCl}, \mathrm{NaCl}$, or $\mathrm{KCl}$. The corresponding binary systems are known to form low melting eutectics ${ }^{18}$ whereby the melting points are further lowered by the addition of water to enable "hydrothermal" processing.

The elemental composition of the as-synthesized samples together with the Brunauer-Emmett-Teller (BET) surface areas are summarized in Table 1:

Typical scanning electron (SEM) and transmission electron microscopy (TEM) micrographs of the resulting samples prepared from the eutectics are shown in Fig. 1. The materials are composed of primary rough carbon frazzles in the $10 \mathrm{~nm}$ range, which are interconnected to give a porous, aerogel-like structure with extended pore transport systems. Interestingly, the morphology on the mesoscale does only weakly depend on the secondary salt added, but is mainly controlled by the presence of $\mathrm{Zn}^{2+}$. This fine particle morphology already indicates very effective surface stabilization of the material throughout the synthesis under hypersaline conditions. Note that the hydrothermal carbonization of glucose without the presence of a salt template generally results in carbonaceous particles that are several orders of magnitude larger than those observed here (approximately $200 \mathrm{~nm}$ and larger). ${ }^{1}$

Compared to the previous work dealing with the introduction of surface areas into hydrothermal carbons, these samples

Table 1 Elemental composition and surface area of the materials. Notation corresponds to synthesis conditions (see the Materials and methods section)

\begin{tabular}{|c|c|c|c|c|c|}
\hline \multirow[b]{2}{*}{ Entry } & \multirow[b]{2}{*}{ Sample } & \multicolumn{3}{|c|}{$\begin{array}{l}\text { Elemental analysis } \\
{[\mathrm{wt} \%]}\end{array}$} & \multirow{2}{*}{$\begin{array}{l}\text { BET surface area } \\
{\left[\mathrm{m}^{2} \mathrm{~g}^{-1}\right]}\end{array}$} \\
\hline & & $\mathrm{C}$ & $\mathrm{N}$ & $\mathrm{H}$ & \\
\hline 1 & $\mathrm{C}-\mathrm{Na} 7 / 3$ & & & & At detection limit \\
\hline 2 & $\mathrm{C}-\mathrm{Na} 7 / 0$ & & & & At detection limit \\
\hline 3 & $\mathrm{C}-\mathrm{Zn} 9 / 3$ & & & & 477 \\
\hline 4 & $\mathrm{C}-\mathrm{Zn} 9 / 0$ & & & & 443 \\
\hline 5 & $\mathrm{C}-\mathrm{Zn} 9 / 14$ & 64.7 & - & 4.7 & Small \\
\hline 6 & $\mathrm{CN}-\mathrm{Zn} 9 / 3$ & 70.0 & 3.0 & 5.0 & 576 \\
\hline 7 & C-LiZn15/3 & 68.2 & - & 4.8 & 673 \\
\hline 8 & C-NaZn13/3 & 68.1 & - & 4.9 & 546 \\
\hline 9 & $\mathrm{C}-\mathrm{KZn} 14 / 3$ & 67.3 & - & 5.0 & 425 \\
\hline
\end{tabular}




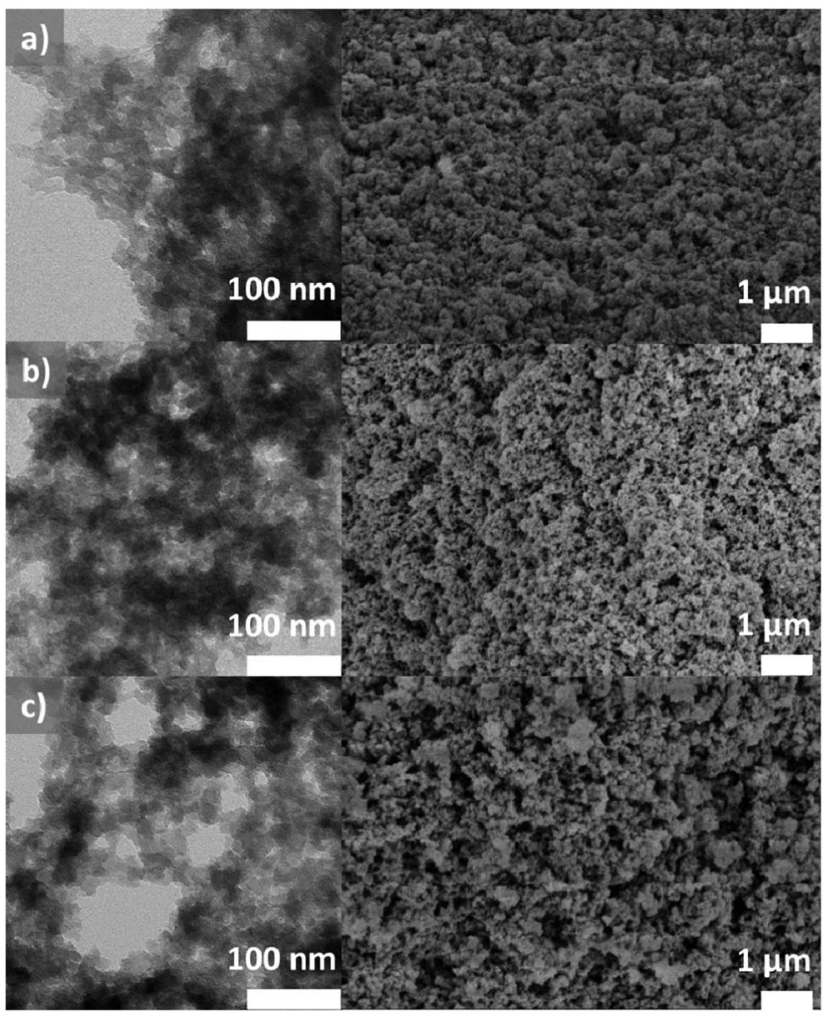

Fig. 1 TEM (left) and SEM (right) pictures of (a) C-LiZn15/3, (b) C-NaZn13/3 and (c) C-KZn14/3.

constitute smaller particles than the albumin-derived aerogels and are comparable to the very best borax-mediated aerogel samples. It must be emphasized that contrary to the previous work, we did not apply critical drying to isolate the samples, that is the porous scaffolds are also stable enough to withstand the high capillary pressures occurring throughout water drying. This is a very rare behavior, even hardly found for silica aerogels which are also preferentially dried in a supercritical fashion. The related nitrogen isotherms are shown in Fig. 2.

All three curves are characterized by the typical surface nitrogen sorption, with the interstitial pores between the particles just starting to be visible in the higher pressure range. The medium relative pressure range is similar for all three

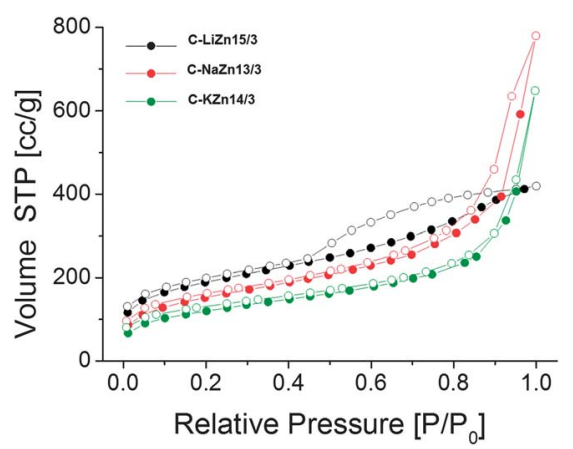

Fig. 2 Nitrogen sorption isotherms of C-LiZn15/3 (black), C-NaZn13/3 (red) and C-KZn14/3 (green). samples, supporting the observations from electron microscopy. Interestingly and very unusual for low temperature hydrothermal carbons, we also find distinct microporosity which is most pronounced for the $\mathrm{ZnCl}_{2} / \mathrm{LiCl}$ sample. This observation points towards imprinting of a simple salt, presumably zinc and lithium entities, into the surface of the particles. It is an exciting question if this imprinted salt would also be recognized in a later rebinding event, but this is not a subject of the present contribution. Concomitantly, a less pronounced gas uptake at higher relative pressures is observed for the $\mathrm{ZnCl}_{2} / \mathrm{LiCl}$ sample indicating smaller interstitial room which is probably due to smaller particles. The as-obtained crude hydrophilic carbonaceous material could further be transferred into the corresponding rather hydrophobic carbons by post-calcination at elevated temperatures, also leading to a significantly enhanced surface area as a result of further water elimination. ${ }^{19,20}$ This would also increase material conductivity and open the doors toward electronic applications as has been previously shown for hydrothermal carbons. ${ }^{21}$

The elemental composition (Table 1) of the samples corresponds very much to a typical HTC scenario: all samples are formed through condensation towards a highly hydrophilic scaffold, the surface of which is saturated with $\mathrm{C}=\mathrm{O}$ and $\mathrm{C}-\mathrm{OH}$ groups as commonly observed by solid state NMR, XPS or FT-IR for standard HTC carbon. ${ }^{1}$ However, the dark brown to black color of the carbons synthesized in hypersaline medium (in contrast to the otherwise light brown materials derived from glucose under standard HTC conditions), together with the increased carbon content points to a higher degree of aromatization.

Since heteroatom-doped carbons are known to show improved performance in a variety of applications, e.g. oxygen reduction reaction (ORR), ${ }^{22,23}$ supercapacitors, and $\mathrm{CO}_{2^{-}}$ capture, $^{24,25}$ and as a proof of principle, we used 2-pyrrol-carboxaldehyde (PCA) as an additive in order to introduce structural nitrogen into the carbon structure. ${ }^{16}$ Concerning elemental analysis and sorption measurements (Table 1), the formation of a very similar structure can be assumed. Yet, the carbon content in the sample prepared with PCA is even higher compared to the ones without an additive, underlining the better aromatization of N-containing precursors in internal Diels-Alder schemes. ${ }^{26}$ This delineates the generality of the approach and opens the way toward highly porous, aerogel-like carbonaceous and nitrogencontaining materials via a convenient, one-step HTC process. Preliminary experiments using those carbons as a support for electrocatalysis indeed show the suitability of those structures for monolith electrochemistry.

\section{Conclusion}

Water containing eutectic salt melts were used as a reaction medium for the hydrothermal carbonization of glucose. In the presence of appropriate hygroscopic salts, e.g. $\mathrm{ZnCl}_{2}$, and only a little water, porous carbonaceous structures with a typical "aerogel" structure and surface areas of $400-650 \mathrm{~m}^{2} \mathrm{~g}^{-1}$ were obtained. It is underlined that the samples were obtained after simple washing with water and ordinary drying, i.e. no 
supercritical $\mathrm{CO}_{2}$ drying was necessary to prevent collapse of the nanostructured carbon aerogels due to capillary forces. This means that the as-formed structures are unusually robust and well cross-linked. It was shown that both the particle size and hence the specific surface area as well as the nitrogen content can be varied by the starting products and the salt mixtures used.

This work therefore demonstrates the powerful potential of HTC also under "geomimetic" hypersaline conditions to synthesize carbon-based porous materials for energy- and water-related applications in a fully sustainable fashion using processes with complete recycling loops and renewable resources.

\section{References}

1 M. M. Titirici and M. Antonietti, Chem. Soc. Rev., 2010, 39, 103-116.

2 B. $\mathrm{Hu}, \mathrm{K}$. Wang, L. H. Wu, S. H. Yu, M. Antonietti and M. M. Titirici, Adv. Mater., 2010, 22, 813-828.

3 A. C. Pierre and G. M. Pajonk, Chem. Rev., 2002, 102, 42434266.

4 J. Biener, M. Stadermann, M. Suss, M. A. Worsley, M. M. Biener, K. A. Rose and T. F. Baumann, Energy Environ. Sci., 2011, 4, 656-667.

5 G. M. Pajonk, Appl. Catal., 1991, 72, 217-266.

6 S. S. Kistler, Nature, 1931, 127, 741.

7 J. L. Mohanan, I. U. Arachchige and S. L. Brock, Science, 2005, 307, 397-400.

8 Y. Shi, Y. Wan and D. Zhao, Chem. Soc. Rev., 2011, 40, 3854.

9 H. Fan, H. R. Bentley, K. R. Kathan, P. Clem, Y. Lu and C. J. Brinker, J. Non-Cryst. Solids, 2001, 285, 79.

10 D. H. Long, J. Zhang, J. H. Yang, Z. J. Hu, G. Cheng, X. M. Liu, R. Zhang, L. Zhan, W. M. Qiao and L. C. Ling, Carbon, 2008, 46, 1259-1262.
11 G. C. Ruben and R. W. Pekala, J. Non-Cryst. Solids, 1995, 186, 219-231.

12 R. W. Pekala, J. Mater. Sci., 1989, 24, 3221-3227.

13 Y. Zhai, Y. Dou, D. Zhao, P. F. Fulvio, R. T. Mayes and S. Dai, Adv. Mater., 2011, 23, 4828.

14 R. J. White, N. Yoshizawa, M. Antonietti and M.-M. Titirici, Green Chem., 2011, 13, 2428-2434.

15 T.-P. Fellinger, R. J. White, M. M. Titirici and M. Antonietti, Adv. Funct. Mater., 2012, 22, 3254-3260.

16 S.-A. Wohlgemuth, T.-P. Fellinger, P. Jäker and M. Antonietti, J. Mater. Chem. A, 2013, 1, 4002-4009.

17 N. Fechler, T.-P. Fellinger and M. Antonietti, Adv. Mater., 2013, 25, 75-79.

18 G. J. Janz, C. B. Allen, J. R. Downey Jr and R. P. T. Tomkins, National Standard Reference Data System, New York, 1978.

19 X. Zhu, P. C. Hillesheim, S. M. Mahurin, C. Wang, C. Tian, S. Brown, H. Luo, G. M. Veith, K. S. Han, E. W. Hagaman, H. Liu and S. Dai, ChemSusChem, 2012, 5, 1912.

20 N. Das, P. Pattanaik and R. Das, J. Colloid Interface Sci., 2005, $292,1$.

21 L. Zhao, L.-Z. Fan, M.-Q. Zhou, H. Guan, S. Qiao, M. Antonietti and M.-M. Titirici, Adv. Mater., 2010, 22, 5202-5204.

22 W. Yang, T.-P. Fellinger and M. Antonietti, J. Am. Chem. Soc., 2011, 133, 206.

23 R. Liu, D. Wu, X. Feng and K. Müllen, Angew. Chem., Int. Ed., 2010, 49, 2565.

24 X. Zhu, P. C. Hillesheim, S. M. Mahurin, C. Wang, C. Tian, S. Brown, H. Luo, G. M. Veith, K. S. Han, E. W. Hagaman, H. Liu and S. Dai, ChemSusChem, 2012, 5, 1912.

25 V. Chandra, S. U. Yu, S. H. Kim, Y. S. Yoon, D. Y. Kim, A. H. Kwon, M. Meyyappan and K. S. Kim, Chem. Commun., 2012, 48, 735.

26 L. Zhao, N. Baccile, S. Gross, Y. Zhang, W. Wei, Y. Sun, M. Antonietti and M.-M. Titirici, Carbon, 2010, 48, 3778. 